\title{
Biomimetic Knee Design to Improve Joint Torque and Life for Bipedal Robotics
}

\author{
Alexander G. Steele ${ }^{1}$, Alexander Hunt ${ }^{1}$, Appolinaire C. Etoundi ${ }^{2}$ \\ ${ }^{1}$ Department of Mechanical Engineering, Portland State University (PSU) Portland, Oregon, \\ United States of America \\ 2 Department Engineering Design and Mathematics, Bristol Robotics Laboratory, University of \\ the West of England, Bristol, United Kingdom \\ ajmar@pdx.edu, ajh26@pdx.edu, Appolinaire.Etoundi@uwe.ac.uk
}

\begin{abstract}
This paper details the design, construction, and performance analysis of a biologically inspired knee joint for use in bipedal robotics. The design copies the condylar surfaces of the distal end of the femur and utilizes the same crossed four-bar linkage design the human knee uses. The joint includes a changing center of rotation, a screw-home mechanism, and patella; these are characteristics of the knee that are desirable to copy for bipedal robotics. The design was calculated to have an average sliding to rolling ratio of 0.079 , a maximum moment arm of 2.7 inches and a range of motion of 151 degrees. This should reduce wear and perform similar to the human knee. Prototypes of the joint have been created to test these predicted properties.
\end{abstract}

Keywords: Condylar Hinge Joint, Knee Biomechanics, Bipedal Robot, Adaptive Robotics, Knee Joint, Screw-Home Mechanism, Four bar linkages

\section{Introduction}

Current articulated robotics systems use common rotational joining techniques through the use of revolute joints, also known as hinge or pin joints. There are several different examples of these joints, but they all have similar properties [1]. This class of joints has a single degree of freedom, rotation about the pin. Typically, the two rigid parts have a pinned center that enables the rotation of the joint. Due to their simplicity to elaborate and drive, the single degree of freedom offered by pin joints makes them desirable for control purposes. Additionally, they offer a low cost and easy to replace option.

However, due to the high shear stress that can be applied to the joint through normal operations, the joints wear quickly [1]. Clamping forces applied to the pin, or preload, also causes joint fatigue, which can lead to premature wearing as it introduces tensile forces, and can induce creep. At the hole, where bearing forces apply and stress concentration occur, the ratio between the diameter of that hole and the edge of the material to the edge of the hole has a direct effect on the wear and failure of the joint. Furthermore, since the joint is a single pin without added supports to strengthen its overall structure, which leads it to cause serious damage within the system it, is placed in when failure occur. 
In recent years, biomimetic design has led to several different attempts to model and design a mechanical joint similar to the human knee like the one shown in Fig 1. These designs have looked at different aspects of the knee and attempted to mimic them. Several of these designs include a screw-home mechanism and patellar analogs [2-6].

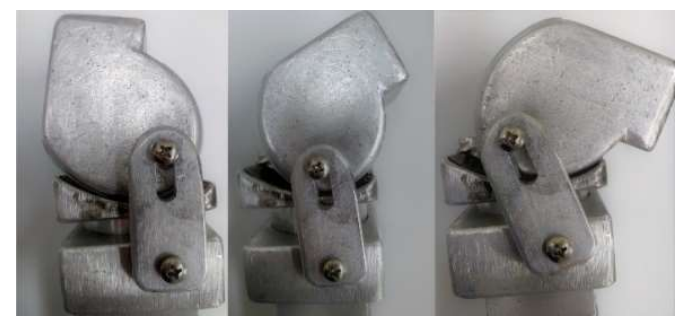

Fig. 1. Previous biomimetic knee design [7]

For most of these designs, the biomimetic approach involves the utilization of the fourbar linkage design when modeling the human knee. In theory, this design should have kinematics close to the human knee. However, the designs that have been put forth are "floating designs" or complex linkage designs that do not have contacting surfaces such their human counterparts [8]. This predominately places load onto the pin connections and while the force profile over the entire range of motion differs, the pin joints have to carry the weight just as the solitary pin joint design first mentioned.

Hobon et al. [9] modeled the benefits of using what they termed as a rolling knee (RK). This joint, shown in Fig. 2, consists of two rolling cylinders joined by a solid link, which has a pin joint at the center of either cylinder. This design eliminates a portion of the force placed on the pin joint by applying it through the contact point of the cylinders. The RK design was developed to be a better solution than pin joints in regards to energy consumption during walking gait.

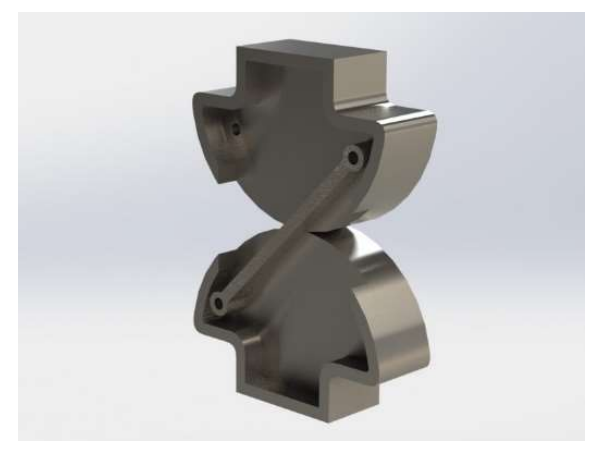

Fig. 2. Rolling knee (RK) model [9]

While the authors state this is a quasi-optimal solution for energy consumption in a walking gait, wear of the joint has not been addressed. By using the RK solution, the 
force from the weight of the robot is applied at a one-dimensional line across the cylinder, this force can cause premature wearing, and an impact load could lead to permanent deformation of the cylinders. Since there is no locking mechanism, energy is needed to keep the robot upright when standing. When fully flexed, the weight of the robot is still applied to the pin joints used to attach the two cylinders, which carries the same problems as the previously discussed pin joint design.

All of these designs fail in some way to maximize joint life because they still place weight primarily on pinned connections. We propose that further analysis of the human knee kinematics and biology can further improve these designs and lead to a better design.

\section{Anatomy And Physiology Of The Human Knee}

The knee joint is significantly more complex than initial observations would reveal. It is also the largest joint in the body. While the motion of the knee looks simple, it has a degree of freedom of six, three rotational and three translational shown in Fig. 3, its movement is constrained by four main ligaments. Although there is some translation occurring in the knee, it is not significant and the dominating movements are rotational: these are flexion/extension, medial rotation, and lateral rotation. The range of motion of the knee during flexion and extension has been found to be between 120 and 150 degrees, with about 10 degrees of rotation medially when the knee is flexed and about 30 to 40 degrees of lateral rotation [10].

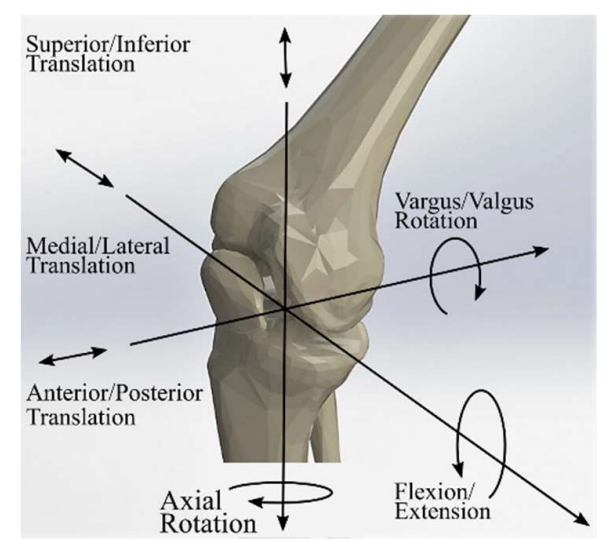

Fig. 3. The degree of freedom is 6 for the human knee [8]

When fully extended, the knee has a locking mechanism called the screw home mechanism. When the tibial head rotates relative to the distal end of the femur, an oblique pull of the ligaments during the last 30 to 35 degrees of extension increase joint stability, resisting forces applied laterally to the body. Once in this locked position, the knee requires little external force to remain locked and in the fully extended position. 
At the knee joint, where the femur and tibia meet, sits the patella (also known as the kneecap) that protects the articular surfaces. The primary role of the patella is assisting in knee extension by increasing the angle in which the patellar tendon acts. This increases the leverage the tendon can exert on the joint by 20 to 30 percent $[11,12]$. The quadriceps tendon attaches the top of patella to the quadriceps femoris muscle, the main muscle responsible for extension of the knee. To resist lateral dislocation, the patella is stabilized by the large size of the lateral femoral that can be seen in Fig. 4.

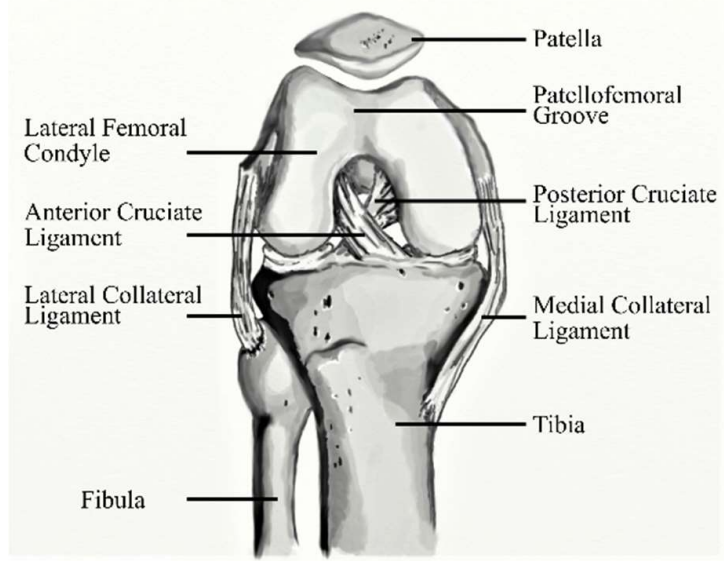

Fig. 4. Anatomy of the human left knee as seen from the front bent at 90 degrees. The patella resists dislocation due in part to the patellofemoral groove. [13]

While the knee has six main ligaments, the focus of this work is on the anterior cruciate ligament (ACL) and the posterior cruciate ligament (PCL). The ACL and PCL are two bundles of bands made up of dense connective tissue located intracapsular to the joint. The ACL resists anterior tibial translation and rotational loads, while the PCL resists posterior translation and shearing forces.

\section{$3 \quad$ Methods: Knee Development}

\subsection{Instantaneous Center of Rotation of the Patellofemoral Joint}

Our design utilizes the same four-bar cross-link configuration found in the human knee so determining the location of the instantaneous center of rotation (ICR) is important because it determines the length of the moment arm. The further away the instantaneous center of rotation is from the line of action produced by the muscle, the less force required to produce the needed amount of torque to create movement.

Figure 5 shows the linkage configuration used in the construction as well as the link designation, angle designation, and coordinate system for the following equations.

For $\mathrm{n}$ number of moving bodies, there are $\mathrm{N}$ number of instantaneous centers of rotation such that 


$$
N=\frac{n(n-1)}{2} \text {. }
$$

From Equation 1, a four-bar linkage has six ICR, four of which are the joints where two links meet. This leaves two ICR that are not explicitly formed at joints; however, we are only interested in one of these ICR. This is the ICR formed by the intersection of the lines created from links 1 and 3, where link 1 is the PCL, link 3 represents the ACL, and links 2 and 4 represent the fibular end and tibial head respectively; this closed link configuration can be defined as four vectors in a closed polygon where the sum is zero, written as

$$
\overrightarrow{A B}+\overrightarrow{B C}+\overrightarrow{C D}+\overrightarrow{D A}=0
$$

However, because $\overrightarrow{D A}$ is parallel to the origin it can be reduced to just the length $\mathrm{L}_{4}$. Then vector equation may be rewritten in complex-number form such that

$$
L_{1} e^{i \theta_{1}}+L_{2} e^{i \theta_{2}}+L_{3} e^{i \theta_{3}}+L_{4}
$$

Next Equation 3 is separated into the real and imaginary parts

$$
\begin{gathered}
L_{1} \cos \left(\theta_{1}\right)=L_{3} \cos \left(\theta_{4}\right)+L_{2} \cos \left(\theta_{3}\right)+L_{4} \\
L_{3} \sin \left(\theta_{1}\right)=L_{3} \sin \left(\theta_{4}\right)+L_{2} \sin \left(\theta_{3}\right)
\end{gathered}
$$

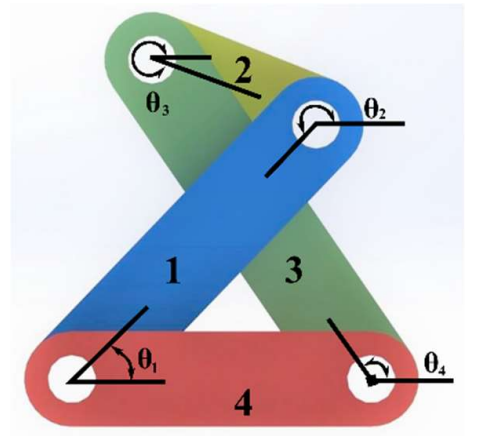

Fig. 5. Four-bar linkage used in the proposed design

Solving to find the intersection of the two links, we obtain the set of equations for the instantaneous center of rotation given in $\mathrm{x}$ and $\mathrm{y}$ coordinates

$$
\begin{aligned}
& x=L_{4} \frac{\tan \left(\theta_{4}\right)}{\tan \left(\theta_{4}\right)-\tan \left(\theta_{1}\right)} \\
& y=L_{4} \frac{\tan \left(\theta_{4}\right) * \tan \left(\theta_{1}\right)}{\tan \left(\theta_{4}\right)-\tan \left(\theta_{1}\right)}
\end{aligned}
$$




\subsection{Design modification for screw-home mechanism analog}

Another important feature of the knee is the screw-home mechanism. An analog to the screw-home mechanism is achieved using the geometry of the linkage because the design uses a solid and crossed 4-bar linkage design. Figure 6 shows how by limiting the forward movement of link 1 through modification of the condylar surface of the tibial head creates a mechanical stop that prevents any additional forward movement.

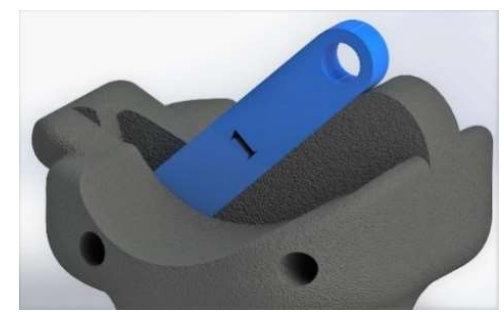

Fig. 6. Modification of the proposed tibial head preventing flexion or over extension when forces are applied superiorly

Once the joint is in the upright position, the modification to the tibial head creates a locking mechanism such that any amount of loading force applied to the knee - for example a standing position - cannot induce a rotation of the joint due to the placement of the ICR at full extension. The design directs weight through the condylar surfaces of the distal end of the femur and the tibial head instead of the connecting links of the joint. This broad surface contact spreads out the load and reduces this source of premature wear and failure.

\subsection{Patellar design for effective moment arm increase}

One of the first considerations for approximating the patella in the design was the contact faces. Contact needs to be maximized to reduce the wear of the surfaces. Additionally, in order to maximize the torque about the joint applied by the quadriceps muscles the force vector applied by the patella to the joint should point perpendicularly to the moment arm. Figure 7 shows how the forces are applied to the knee joint, where the resultant force $\mathrm{F}(\theta)$ passes through the instantaneous center of rotation and is calculated by

$$
F(\theta)=2 Q * \cos \left(\frac{\theta}{2}\right)
$$

The angle $\theta$ is the angle between the quadriceps tendon and the patellar tendon. Because the ICR curve of the designed joint is flattened, the resultant force can be directed perpendicularly to the moment arm and through the ICR longer than an ICR curve that was more convex. By correctly angling the patellar using Equation 8 , the resultant force from the actuator can be maximized through the entire extension of the joint. 


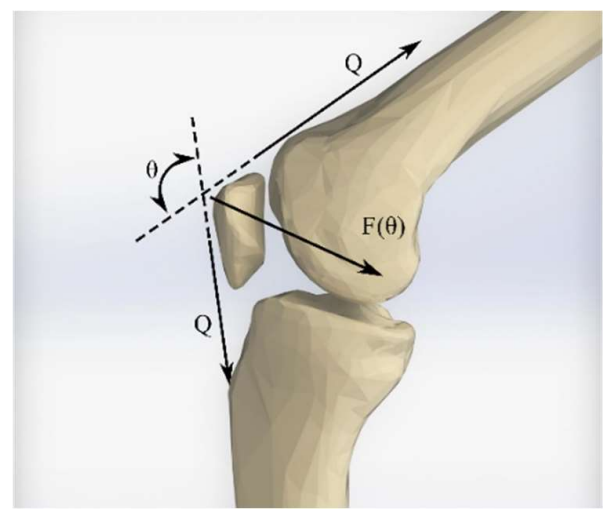

Fig. 7. Force applied to the knee through the patella as seen from the side

\subsection{Designing the condylar surfaces to ensure contact throughout joint motion}

Design of the distal end of the femur and the tibial head were done to ensure that the condylar surfaces made contact throughout the entire range of motion of the joint. By doing this we can redirect the forces from the linkage to the condylar surfaces, this will reduce wear and reduce the risk of catastrophic failure.

However, because the linkages we are using are solid and the linkages of the human knee are flexible, the contacting surfaces of the knee could not be copied outright; instead a new design for the tibial contact surfaces had to be designed to reduce sliding while still being compact. For that reason, the curve of the medial condyle, the larger of the two condyles, was chosen for both the contact surfaces of the distal end of the femur.

The tibial head had to be designed so that when the end of the femur is constrained to the crossed four-bar linkage it maintained contact throughout the range of motion. In order to do this, the shape of the curve created by the femur when constrained by the four-bar linkage was calculated and then used to create a 2-dimensional profile that would be used to create the contact surfaces of the tibia.

This curve was then turned into the profile of the tibial contact surface using SolidWorks. The rest of the tibial head was then designed around the movement of the linkage so that it would not interfere with the range of motion.

\subsection{Modification of the tibial head to eliminate deflection from out-of-plane forces}

When using the basic four-bar link design, out-of-plane forces applied at full flexion caused unwanted twisting and bending to the joint. This bending puts the linkages in a high stress condition, which could cause premature wear or failure. 
To eliminate this, an extension was added and designed to lock into the distal end of the femur, which can be seen in Figure 8. This addition removes the force applied to the links at full flexion, applying it directly to the back of the tibial head.

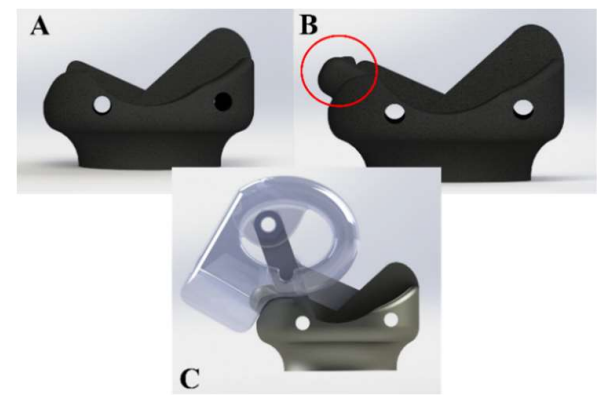

Fig. 8. Tibial head seen laterally (A) prior to modification, (B) post modification, change highlighted in red circle, and (C) tibial head locked with the distal end of the femur for reference.

\subsection{Joint sliding-rolling ratio}

To maximize the life of the joint, it is desirable to have a sliding- rolling ratio as close to zero as possible, this would indicate that the joint is in a pure rolling condition. Alternatively a sliding to rolling ratio of one would indicate that the joint is in a pure sliding condition, which leads to premature wear [14]. Using SolidWorks, we created a motion study to determine the sliding to rolling ratio of the joint. Figure 9 shows how the ratio is determined. First, the travel arc length of the tibia $\left(\Delta \mathrm{L}_{\mathrm{T}}\right)$ and the travel arc length of the femur $\left(\Delta \mathrm{L}_{\mathrm{F}}\right)$ are defined as

$$
\begin{aligned}
& \Delta L_{T}(\theta)=C_{T f}(\theta)-C_{T i}(\theta) \\
& \Delta L_{F}(\theta)=C_{F f}(\theta)-C_{F i}(\theta)
\end{aligned}
$$

Where $\mathrm{C}_{\mathrm{Ti}}$ and $\mathrm{C}_{\mathrm{Fi}}$ are the initial contact points and $\mathrm{C}_{\mathrm{Tf}}$ and $\mathrm{C}_{\mathrm{Ff}}$ are the final contact points of the tibia and femur given an incremental flexion angle $(\theta)$. Once the travel arc length is calculated, the sliding to rolling ratio (SR) is calculated by

$$
S R(\theta)=\frac{\Delta L_{T}(\theta)-\Delta L_{F}(\theta)}{\Delta L_{T}(\theta)}
$$

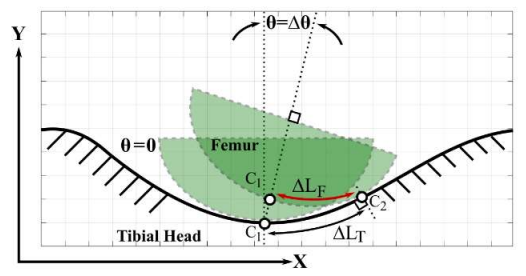

Fig. 9. Kinematics of sliding to rolling ratio from initial contact point $C_{1}$ to $C_{2}$ 


\subsection{Joint Construction}

Figure 10 shows the physical joint that will be used for future testing. Construction of this test joint was done using a $3 \mathrm{D}$ printer that has the ability to inlay continuous carbon fiber into the print. This imbues the part with exceptional strength. Metal fasteners were placed directly into the print so that metal shafts and lubricated sleeve bearings could be added to reduce friction at the joint connections. By adding the fasteners directly into the print, a clamping force is not required to keep the link on the shaft. This ensures that the link cannot physically become separated from the shaft and eliminates one factor of joint wear.

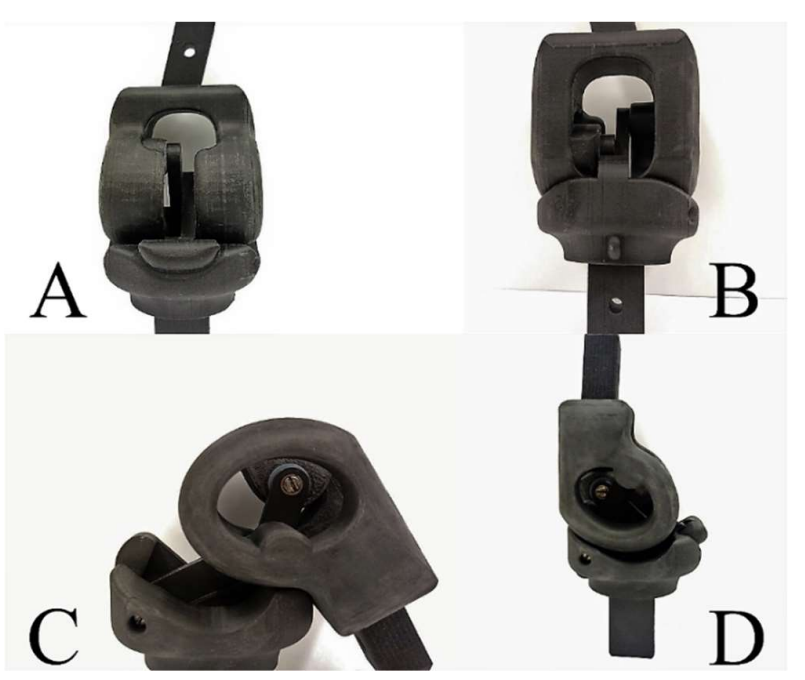

Fig. 10. Constructed joint (A) rear view, (B) front view, (C) full flexion, and (D) full extension

\section{$4 \quad$ Results}

\subsection{Instantaneous center of Rotation}

The length of the links which correspond to our designed joint are [L1, L2, L3, L4] $=[1.85,0.92,2.03,1.57]$ given in inches. A plot of the changing moment arm as the knee as it goes from fully extended to fully flexed is shown in Figure 11. The ICR only moves \pm 0.2 inches out of plane from the desired line of action and has a maximum overall moment arm of 2.7 inches prior to the addition of a patella.

The designed joint is only 2.8 inches wide and has a range of motion of 151 degrees; this roughly the same size and range of motion of the human knee. In a fully extended position, such as when standing, the joint locks so that no extra energy is needed to keep the joint in that position, this feature in conjunction with the large moment arm should greatly reduce the energy requirements of a robot when compared to one with a pin joint. 


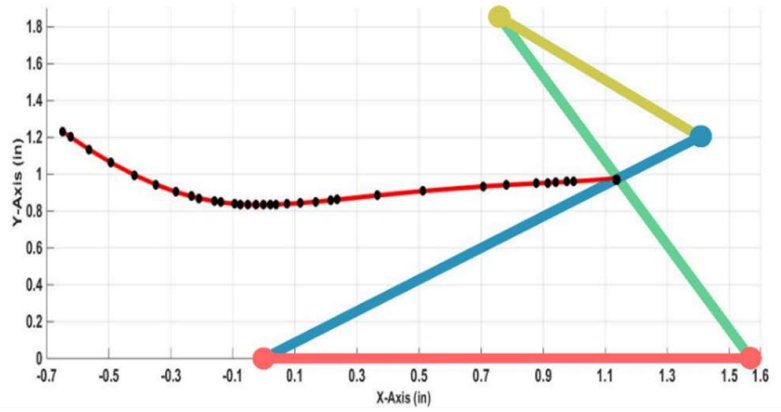

Fig. 11. Instantaneous center of rotation plot of proposed joint for the full range of motion.

\subsection{Sliding to Rolling Ratio}

Figure 12 is the SolidWorks Motion Analysis results for the sliding to rolling simulation with regard to flexion calculated at 0.5 -degree intervals through the full range of motion of the joint. The ratio of sliding to rolling of the proposed joint, on average is 0.079 with a minimum value of 0.018 and a maximum value of 0.28 . Because a sliding to rolling ratio close to zero is desirable for wear, the low average and maximum values should give the joint an exceptionally high lifetime when compared to revolute joints.

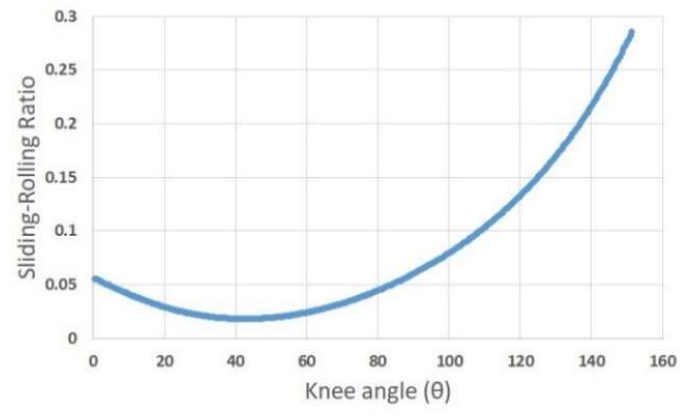

Fig. 12. Sliding to rolling ratio over the designed joints range of motion where 0 is a pure rolling condition and 1 is a pure sliding condition, which increases wear.

\section{Discussion}

Comparison of the ICR curve with one developed by Fekete et al. [15] demonstrate that the placement of the curve is similar to the placement of the ICR curve of the human knee. Due to the compliance of tendons in the human knee, unlike the solid links used in the current design, the ICR curve of a human knee is more convex than the ICR 
curve of the designed knee. However, the placement of the curve is similar to the placement of the ICR curve of the human knee and the flattened ICR curve of the designed joint has desirable properties with respect to the force requirements to create motion.

There are too many variables to determine the sliding to rolling ratio of the human knee in vivo so we cannot confidently include a graphical comparison. However, current research on the subject suggests the sliding to rolling ratio of the human knee is between 0.3-0.46 [8, 14].

By mimicking the design of the human knee, the designed joint is comparable in size while having several of the knees' desirable properties. This is in part due to the complex geometry that was obtained from 3D printing instead of using traditional methods. $3 \mathrm{D}$ printing also reduced the time of construction to just over one day as compared to potentially weeks of traditional machining.

Because the joint is made primarily from a nylon and carbon fiber chop and has a continuous carbon fiber inlay, the joint has exceptional strength and can be used functionally, but weighs just 0.66 pounds. Furthermore, the majority of the weight of the joint is due to the steel fastening hardware added internally to the design mid-print and not from the print material itself.

One of the goals in the design of our knee joint was to reduce wear, with an average sliding to rolling ratio of 0.079 , the joint has a significantly lower sliding to rolling ratio than the human knee. By having continuous contact of the loading faces, the load applied to the pins is minimized and the addition of the locking extension on the rear of the tibia transfers a majority of the forces that would be placed on the pins at full flexion. By removing most of the loading forces from the pin connections, the joint will be less likely to suffer from a catastrophic failure and should last significantly longer than the traditional pin joint.

\section{Acknowledgments}

The authors acknowledge the support of the Mechanical and Materials Engineering Department in the Maseeh College of Engineering at Portland State University and the support of the UK Engineering and Physical Sciences Research Council (EPSRC) under grant reference EP/P022588/1.

\section{References}

1. Saad Mukras, Nam H. Kim, Nathan A. Mauntler, Tony L. Schmitz, W. Gregory Sawyer: Analysis of planar multibody systems with revolute joint wear. Wear. 268, 643-652 (2010).

2. Asano, Y., Mizoguchi, H., Kozuki, T., Motegi, Y., Urata, J., Nakanishi, Y., Okada, K., Inaba, M.: Achievement of Twist Squat by Musculoskeletal Humanoid with Screw-Home Mechanism. IEEERSJ Int. Conf. Intell. Robots Syst. (2013).

3. Appolinaire C. Etoundi: A bio-inspired condylar hinge joint for mobile robots. Int. Conf. Intell. Robots Syst. (2011). 
4. Burgess, S.C., Etoundi, A.C.: Performance Maps for a Bio-Inspired Robotic Condylar Hinge Joint. J. Mech. Des. 136, 115002-115002-7 (2014).

5. Khan, H., Featherstone, R., Caldwell, D.G., Semini, C.: Bio-inspired knee joint mechanism for a hydraulic quadruped robot. In: 2015 6th International Conference on Automation, Robotics and Applications (ICARA). pp. 325-331 (2015).

6. Etoundi, A., Burgess, S., Vaidyanathan, R.: A Bio-Inspired Condylar Hinge for Robotic Limbs. J. Mech. Robot. 5, 8 (2013).

7. Steele, A.G., Hunt, A., Etoundi, A.C.: Development of a Bio-inspired Knee Joint Mechanism for a Bipedal Robot. In: Biomimetic and Biohybrid Systems. pp. 418-427. Springer, Cham (2017).

8. Masouros, S.D., Bull, A.M.J., Amis, A.A.: Biomechanics of the knee joint. Orthop. TRAUMA. 24, 84-91 (2010).

9. Hobon, M., Elyaaqoubi, N.L., Abba, G.: Quasi optimal sagittal gait of a biped robot with a new structure of knee joint. Robot. Auton. Syst. 62, 436-445 (2014).

10. J. M. Soucie, C. Wang, A. Forsyth, S. Funk, M. Denny, K. E. Roach, D. Boone: Range of motion measurements: reference values and a database for comparison studies. Haemophilia. 500-507 (2011).

11. Alice JS Fox, Florian Wanivenhaus, Scott A Rodeo: The Basic Science of the Patella: Structure, Composition, and Function. J. Knee Surg. 25, 127-141 (2012).

12. Halonen, K.S., Mononen, M.E., Jurvelin, J.S., T€oyr€as, J., Kłodowski, A., Kulmala, J.-P., Korhonen, R.K.: Importance of Patella, Quadriceps Forces, and Depthwise Cartilage Structure on Knee Joint Motion and Cartilage Response During Gait. J. Biomech. Eng. 138, (2016).

13. Jawad F. Abulhasan, Michael J. Grey: Anatomy and Physiology of Knee Stability. J. Funct. Morphol. Kinesiol. 2, (2017).

14. Fekete, G., De Baets, P., Abdel Wahab, M., Csizmadia, B., Katona, G., Vanegas-Useche, L., Solanilla, J.: Sliding-Rolling Ratio during Deep Squat with Regard to Different Knee Prostheses. Acta Polytech. Hung. 9, 20 (2012).

15. Fekete, G.: Kinetics and Kinematics of the Human Knee Joint under Standard and Non-Standard Squat Movement, (2013). 